\title{
Continuous Percolation Transition in Random Cluster Growth Model ${ }^{\dagger}$
}

\author{
Bappaditya Roy and Sitangshu Bikas Santra* \\ Department of Physics, Indian Institute of Technology Guwahati, Guwahati-781039, Assam, India
}

RECEIVED JUNE 24, 2013; REVISED NOVEMBER 22, 2013; ACCEPTED NOVEMBER 26, 2013

\begin{abstract}
A random cluster growth model is developed in terms of two parameters, the initial seed concentration $\rho$ and the growth probability $g$ of individual clusters. The model is studied on a two dimensional square lattice. For every $\rho$ value, a critical value of $g=g_{\mathrm{c}}$ is determined at which a percolation transition is observed. A scaling theory for this model is developed and numerically verified. The scaling functions are found to scale with $\rho, g$ as well as the system size $L$ with appropriate critical exponents. The values of the critical exponents are found to belong to the same universality class of percolation. Finally a phase diagram is developed in the $\rho-g$ parameter space for percolating and non-percolating regions separated by a line of second order phase transition points. (doi: 10.5562/cca2313)
\end{abstract}

Keywords: percolation, random cluster growth, variable initial conditions, continuous transitions, universality class

\section{INTRODUCTION}

Percolation is a model of disordered systems and has extensive applications in different branches of science. For example: oil recovery from porous media, ${ }^{1}$ epidemic modeling, ${ }^{2}$ networks, ${ }^{3,4}$ fracture, ${ }^{5}$ metal-insulator transition, ${ }^{6}$ ionic transport in glasses and composites, ${ }^{7}$ ground water flow in fractured rocks ${ }^{8}$ and many others. Percolation refers to the formation of long-range connectedness in a system and is known to exhibit a continuous phase transition from a disconnected to a fully connected phase at a sharply defined percolation threshold value. ${ }^{9}$ The interest in studying percolation problem in recent time has been triggered by the introduction of a controversial phenomenon called "explosive percolation" (EP).$^{10}$ It had been shown that percolation transition can occur in an explosive manner with a discontinuous jump in the size of the largest cluster above percolation threshold ${ }^{11,12}$ when the growth rules are modified. However, in many cases it were also observed that the transitions are continuous. ${ }^{13,14}$ Recently it is demonstrated analytically as well as numerically that EP transition can be either continuous or discontinuous depending on the bias and the dimensionality of the system. ${ }^{15}$

In this paper a generalized random cluster growth model with different initial seed concentration $\rho$ and tunable growth probability $g$ is presented. For a given $\rho$, a critical growth probability $g_{\mathrm{c}}$ is found to exists at which continuous percolation transition occurs. Such a transition occurs for any value of $\rho$ in the range $1 / L^{2} \leq \rho$ $\leq p_{\mathrm{c}}$ for their corresponding $g_{\mathrm{c}}$ value. All such transitions are found to be characterized by a set of critical exponents same as that of ordinary percolation. No evidence of EP is observed for any value of $\rho$ and $g$. Rather, a line of second order phase transition points in the $\rho-g$ parameter space is found to separate the discontinuous and fully-connected phases.

\section{MODEL}

Consider a two dimensional $(2 d)$ square lattice of size $L$ $\times L$. The lattice is populated randomly with initial seeds with given concentration $\rho$. The seeds may form small clusters of occupied sites randomly distributed all over the lattice. A cluster is a collection of occupied sites connected by nearest neighbour bonds. The clusters are identified by Hoshen-Kopelman algorithm ${ }^{16}$ and labeled as per their size $\mathrm{s}$ and a cluster size distribution function $n_{\mathrm{s}}(\rho)$ is identified. All these clusters are then grown by occupying the empty nearest neighbours (NN) of the perimeter sites of these clusters with probability $g \in[0$, 1] following Leath algorithm. ${ }^{17}$ Once a site is rejected with probability $(1-g)$, it will remain empty forever, as in ordinary percolation. During the growth of these clusters, two clusters may come in contact. Two clusters with occupied perimeter sites separated by a single lattice spacing is considered to be a single cluster. The total number of clusters is then reduced by one and a cluster of larger size is incorporated in the cluster size distribu-

\footnotetext{
$\dagger$ Dedicated to Professor Douglas Jay Klein on the occasion of his $70^{\text {th }}$ birthday.

* Author to whom correspondence should be addressed. (E-mail: santra@iitg.ernet.in)
} 


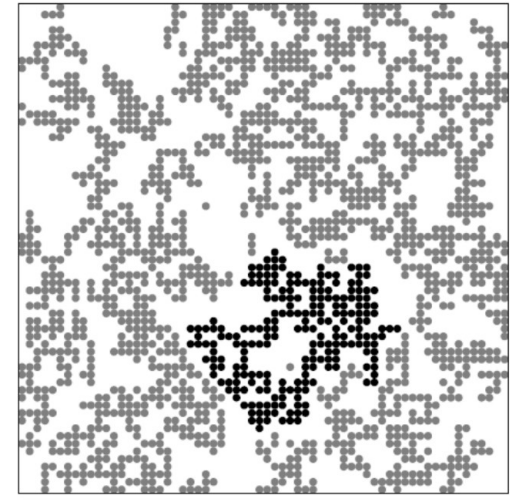

(a)

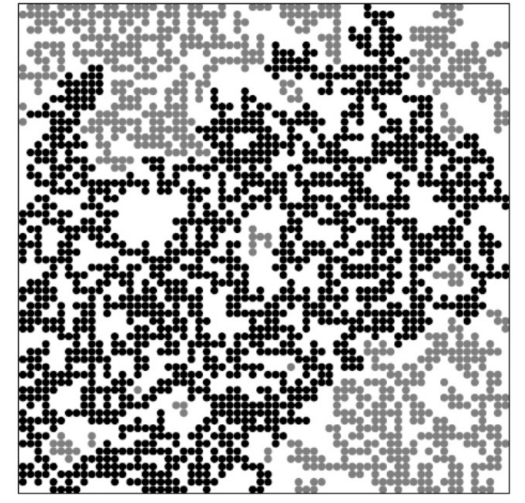

(b)

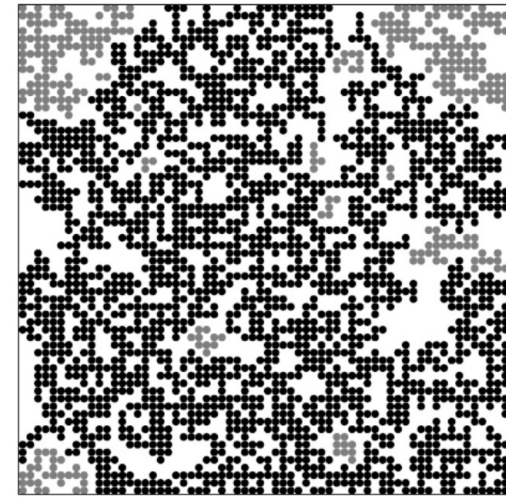

(c)

Figure 1. Snapshots of cluster size distributions at the end of the growth process on a $2 d$ square lattice of size $L=64$ with initial seed concentration $\rho=0.05$ and growth probability $g=0.5,0.57,0.6$. In growing the clusters periodic boundary conditions are used. Solid line represents the lattice boundary. The dark colour indicates the largest cluster. A spanning cluster appears for $g \approx 0.57$.

tion function. The growth of a cluster stops when there is no empty site on the cluster perimeter that is available to occupy. As the process stops, the final cluster size distribution function $n_{\mathrm{s}}(\rho, g)$ is obtained. The model has two limiting situations. One is $\rho=p_{\mathrm{c}}$, the critical site occupation probability of percolation and $g=0$. The other one is $\rho=1 / L^{2}$, a single seed and $g=p_{\mathrm{c}}$. Both situations correspond to ordinary percolation problem. The present model can be considered as a generalized random cluster growth model. It should be noted that the present model is substantially different from the "touch and stop" model. ${ }^{18,19}$ In the later model, the initial clusters also grow with a constant rate (with unit probability) however the growth stops as soon as two clusters come in contact. In the following, taking intermediate values of $\rho$ and varying the growth probability $g$, transitions from disconnected phase to fully connected phase are studied.

Snapshots of the clusters generated on a lattice of size $64 \times 64$ for $\rho=0.05$ and three different values of $g$ $=0.5,0.57,0.6$ are shown in Figure 1. It can be noticed that at $g=0.57$ a spanning cluster appears in the system (Figure 1b). For $g=0.5$ there does not exists any spanning cluster (Figure 1a) whereas for $g=0.6$ the largest cluster occupies most of the space (Figure 1c).

\section{SCALING IN TERMS OF $\rho, g$ AND $L$}

In ordinary percolation, the scaling of a geometrical quantities are studied in terms of area fraction $p$. The cluster size distribution $n_{\mathrm{s}}(p)$ at a given area fraction in the critical regime is assumed to be a generalized homogeneous function of $\left(p-p_{\mathrm{c}}\right)$ and the cluster size $s$ as

$$
n_{\mathrm{s}}(p)=s^{-\tau} f\left[\left(p-p_{\mathrm{c}}\right) s^{\sigma}\right]
$$

where $p_{\mathrm{c}}$ is the critical area fraction or the percolation threshold at which a spanning cluster appears for the first time in the system and $\tau, \sigma$ are two critical exponents. The geometrical quantities can be derived from the above distribution (Eq. 1) as given in Ref. 9. The scaling behaviour of the geometrical quantities are also obtained in terms of $\left|p-p_{\mathrm{c}}\right|$. For example, the probability that a site belong to the spanning cluster scales as $P_{\infty}$ $\approx\left(p-p_{\mathrm{c}}\right)^{\beta}$, the average cluster size $\chi \approx\left|p-p_{\mathrm{c}}\right|^{-\gamma}$, the connectivity length $\xi \approx\left|p-p_{\mathrm{c}}\right|^{-v}$, etc. where $\beta, \gamma, v$ are respective critical exponents. However, in the present problem one starts with an initial seed concentration $\rho$ and the empty sites around the clusters formed by the initial seeds are grown with probability $g$, the area fraction $p$ at the end of the growth process is expected to be

$$
p=\rho+g(1-\rho)
$$

for any system size $L$. Note that for $g=1$, the final area fraction would be always one irrespective of initial seed concentration. For a fixed $\rho$, the difference in area fractions from the threshold value is then given by $p-p_{\mathrm{c}}=(\mathrm{g}$ $\left.-g_{\mathrm{c}}\right)(1-\rho)$. Hence the scaling form of the cluster size distribution and that of all other related geometrical quantities can be obtained in terms of $g$ and $\rho$. The cluster size distribution in terms of $g$ and $\rho$ can be described as

$$
n_{\mathrm{s}}(p, g)=s^{-\tau} f\left[\left(g-g_{\mathrm{c}}\right)(1-\rho) s^{\sigma}\right]
$$

where $f$ is a new scaling function and $\tau, \sigma$ are new scaling exponents. The scaling form of different geometrical quantities in terms of $\rho$ and $g$ can be derived from the above cluster size distribution $n_{\mathrm{s}}(\rho, g)$ in terms of $\rho$ and $g$ as per their definitions in terms of the distribution function.

Below, results obtained from extensive computer simulation are presented. Simulations of the above 

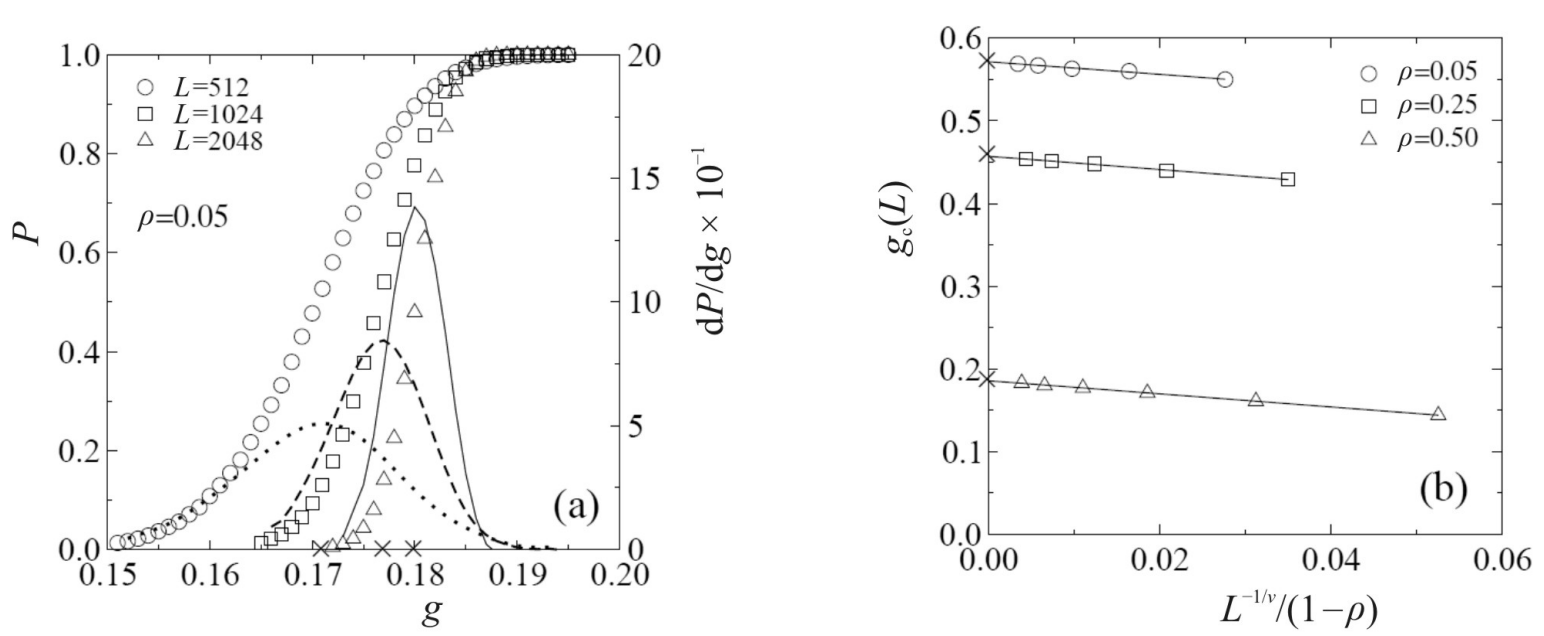

Figure 2. (a) Plot of spanning probability $P$ and its derivative $\mathrm{d} P / \mathrm{d} g$ against $g$ for different system sizes $L$ taking $\rho=0.05$. The symbols are: circle for $L=512$, squares for $L=1024$ and triangles for $L=2048$ and the derivatives are represented by a dotted black line, a dashed lines and a solid line respectively. The values of $g$ corresponding to the maxima of the derivatives indicate $g_{\mathrm{c}}(L)$ and marked by crosses. (b) Plot of $g_{\mathrm{c}}(L)$ versus $L^{-1 / v} /(1-\rho)$ for for $\rho=0.05(\circ), 0.25(\square)$ and $0.50(\Delta)$. The best straight line fit is found for $v=4 / 3$. From the intercepts, $g_{\mathrm{c}}(\rho)$ are obtained as $0.571,0.457,0.186$ respectively.

model are performed for several values of $\rho$ varying the growth probability $g$ on square lattices of size $L$. The size of the system is varied from $L=128$ upto $L=2048$ in multiple of 2 . Clusters are grown applying periodic boundary condition (PBC) in both the horizontal and vertical directions. Ensemble average is made on $10^{5}$ random configurations.

\section{PERCOLATION THRESHOLD}

Percolation threshold is identified as a critical growth probability $g_{\mathrm{c}}$ for a given $\rho$ at which for the first time a spanning cluster connecting the opposite sides of the lattice appears in the system. In order to calculate $g_{\mathrm{c}}$ of a given $\rho$ and system size $L$, the probability to get a spanning cluster

$$
P(\rho, g, L)=\frac{N_{s p}(\rho, g, L)}{N_{t o t}}
$$

in an ensemble of $N_{\text {tot }}$ clusters generated is estimated. For a system size $L \ll \xi, P(\rho, g, L)$ is expected to be

$$
P(\rho, g, L)=f\left[\left(g-g_{\mathrm{c}}\right)(1-\rho) L^{1 / v}\right]
$$

since $L \approx \xi$ and $p-p_{\mathrm{c}}=\left(g-g_{\mathrm{c}}\right)(1-\rho)$. In the $L \rightarrow \infty$, $\mathrm{P}(\rho, g, L)$ is expected to be a theta function at $g=g_{\mathrm{c}}$. Its derivative with respect to $g$ given by

$$
\frac{\mathrm{d} P}{\mathrm{~d} g}=(1-\rho) L^{1 / v} f^{\prime}\left[\left(g-g_{\mathrm{c}}\right)(1-\rho) L^{1 / v}\right]
$$

is a delta function in the $L \rightarrow \infty$ limit with its argument varying from $-\infty$ to $+\infty$. Therefore, for a given $\rho$, the value of $g_{\mathrm{c}}(\rho, L)$ at which a spanning cluster appears for the first time is taken as the average $g$ and can be defined as

$$
g_{\mathrm{c}}(\rho, L)=\int_{0}^{1} g \frac{\mathrm{d} P}{\mathrm{~d} g} \mathrm{~d} g
$$

the average value of $g$ for the distribution of $\mathrm{d} P / \mathrm{d} g$. Sub stituting the value of $\mathrm{d} P / \mathrm{d} g$ from Eq. 6, one may have

$$
g_{\mathrm{c}}(\rho, L)=g_{\mathrm{c}}(\rho) \int_{-\infty}^{+\infty} f^{\prime}(z) \mathrm{d} z+\frac{L^{-1 / v}}{1-\rho} \int_{-\infty}^{+\infty} z f^{\prime}(z) \mathrm{d} z
$$

where $z=\left(g-g_{\mathrm{c}}\right)(1-\rho) L^{1 / v}$. Since in the $L \rightarrow \infty$ limit the function $f(z)$ is a theta function, one has $f(+\infty)=1$ and $f(-\infty)=0$ and the integral $\int_{-\infty}^{+\infty} f^{\prime}(z) \mathrm{d} z=1$. Assuming that $f^{\prime}(z)$ is not a symmetric function, the integral $\int_{-\infty}^{+\infty} z f^{\prime}(z) \mathrm{d} z=C$. where $C$ is a constant. Hence one has

$$
g_{\mathrm{c}}(\rho, L)=g_{\mathrm{c}}(\rho)+C \frac{L^{-1 / v}}{1-\rho}
$$

In Figure 2a, for $\rho=0.05$ the probability of having a spanning cluster $\mathrm{P}(\rho, g, L)$ is plotted against $g$ for three different values of $L, L=512$ (circles), $L=1024$ (squares) and $L=2048$ (triangles). Their derivative are also shown by lines in the same figure. Dotted line 


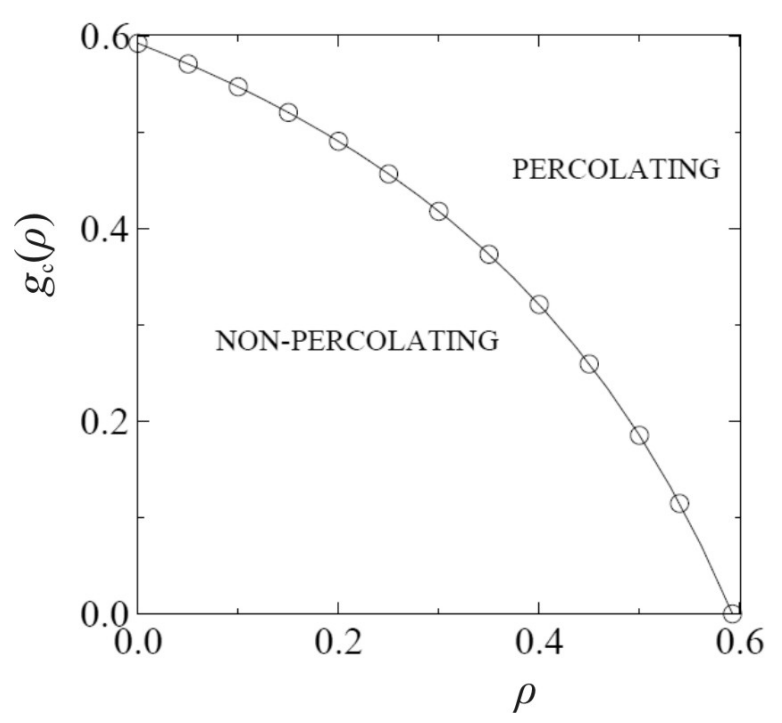

Figure 3. Plot of $g_{\mathrm{c}}(\rho)$ against $\rho$. The circles represent the estimated threshold and the line represents the analytical value obtained from Eq. 10.

represents the derivative for $L=512$, dashed line is that for $L=1024$ and the solid line is that for $L=2048$. The value of $g_{\mathrm{c}}(\rho, L)$ is identified as the value of $g$ corresponding to the maximum of the derivatives and marked by crosses on the $g$-axis. In Figure $2 \mathrm{~b}, g_{\mathrm{c}}(\rho, L)$ are plotted against $L^{-1 / v} /(1-\rho)$ taking $v=4 / 3$ as that of percolation. It has been verified that the best straight line was found for $v=4 / 3$. The percolation threshold $g_{\mathrm{c}}(\rho)$ for infinite system size is then obtained form the intercepts with the $y$-axis. Since the other geometrical properties are evaluated for selective $\rho$ values, the threshold $g_{\mathrm{c}}(\rho)$ for $\rho=0.05,0.25$ and 0.50 are obtained as $0.571 \pm 0.001,0.457 \pm 0.001,0.186 \pm 0.001$ respectively.

\section{PHASE DIAGRAM}

A phase diagram for the percolating and nonpercolating regions on the $\rho-g$ parameter space can be plotted now. Since the percolation has to occur at a well defined threshold value of area fraction $p_{\mathrm{c}}$, the initial seed concentration $\rho$ and the growth probability $g$ must satisfy the following equation

$$
\rho+g_{\mathrm{c}}(1-\rho)=p_{\mathrm{c}}
$$

at the percolation transition. It means that there exists a well defined $g_{\mathrm{c}}$ value for every $\rho$ in order to achieve the critical area fraction $p_{\mathrm{c}}$ at the percolation transition. The critical growth probabilities $g_{\mathrm{c}}(\rho)$ are determined following the method described in section IV for a series of $\rho$ values. The values of $g_{\mathrm{c}}(\rho)$ are plotted against $\rho$ in Figure 3. The solid line represents the Eq. 10. It is es-

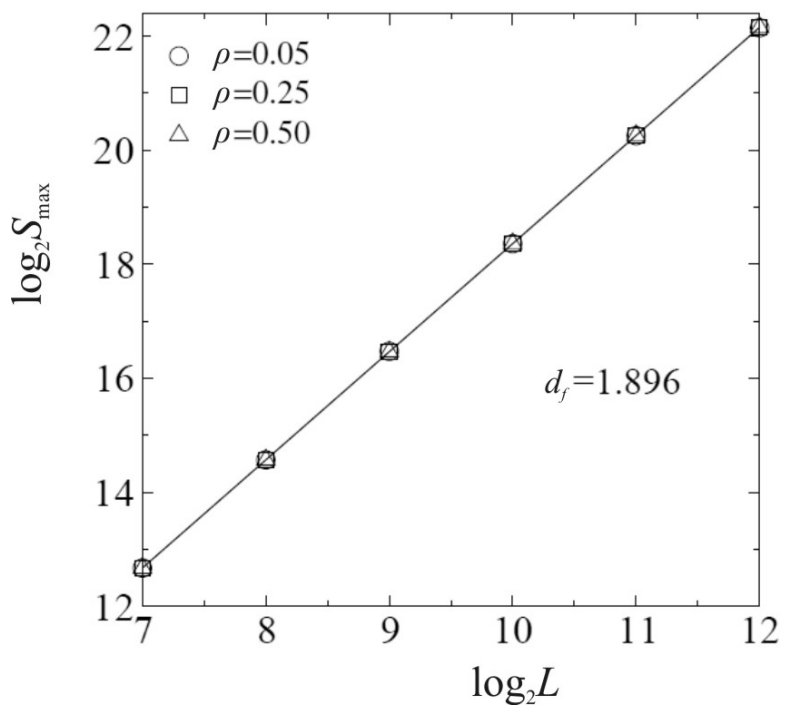

Figure 4. Plot of the spanning cluster size $S_{\max }$ versus $L$ at the percolation threshold $g=g_{\mathrm{c}}(\rho)$ for three different $\rho$ values $0.05(\circ), 0.25(\square), 0.50(\Delta)$. The fractal dimension $d_{f}=1.896$ is estimated from the slope of the straight line and found to be independent of $\rho$.

sentially a line of second order phase transition points which terminates at two trivial percolation transition points. For any $(\rho, g)$ value below this line there will be no percolation transition whereas for any value of $(\rho, g)$ above this line there always be a percolation transition of a first order type. However, at every point on this line there should be a continuous percolation transition. In the following, the universality class of such percolation transitions will be determined and compared with that of ordinary percolation.

\section{FRACTAL DIMENSION OF SPANNING CLUSTER}

Since the clusters are grown here applying PBC, the horizontal and vertical extensions of the largest cluster is kept stored. If either the horizontal or the vertical extension of the largest cluster is found to be $\geq L$, it is identified as a spanning cluster. The percolation spanning cluster is a random object with all possible holes in it and is expected to be fractal. For system size $L<<\xi$, the mass of the spanning cluster varies with the system size $L$ as

$$
S_{\max } \approx L^{d_{f}}
$$

where $d_{f}$ is the fractal dimension of the spanning cluster. The value of $S_{\max }$ is estimated at $g=g_{\mathrm{c}}(\rho)$ for several system sizes and for different values of $\rho$. In Figure $4, S_{\max }$ is plotted against $L$ in double logarithmic scale. A straight line of slope 1.896 is drawn through the data 
points as a guide to eye. It can be seen that the data points follow the straight line with the specified slope. Therefore, the value of the fractal dimension can be taken as $d_{f}=1.896$ correct upto the third decimal place of the exact value $91 / 48$ for the percolation spanning clusters. It is also interesting to note that the values of $S_{\max }$ for different $\rho$ values are not only following the scaling form given in Eq. 11 but also their magnitudes are almost same for a given lattice size. This means that the area fraction given in terms of $\rho$ and $g$ in Eq. 2 holds rightly at the percolation threshold and produced spanning clusters of same size. It could be noted here that in the touch and stop model, ${ }^{19}$ for low concentration of initial seed the final area fraction was found to be same.

\section{CLUSTER SIZE DISTRIBUTION AND GEO- METRICAL QUANTITIES}

In order to determine the scaling behaviour of different geometrical quantities, the cluster size distributions per lattice site $n_{\mathrm{s}}(g, \rho)=N_{\mathrm{s}}(g, \rho) / L^{2}$ are estimated for different values of $g$ and $\rho$. For a given $\rho$, the cluster size distribution is expected to obey a power law scaling behaviour at $g=g_{\mathrm{c}}$ as per Eq. 3 and can be given by

$$
n_{\mathrm{s}}\left(\rho, g_{\mathrm{c}}\right) \approx s^{-\tau}
$$

where $\tau$ is an exponent and it is assumed that $f(0)$ is a constant. For a system size $L=2048, n_{\mathrm{s}}\left(\rho, g_{\mathrm{c}}\right) \mathrm{s}$ are obtained for three different values of $\rho=0.05,0.25,0.50$. The distributions are plotted in Figure 5. The distributions are found to obey power law scaling with an exponent $\tau=2.055(\approx 187 / 91)$ as that of ordinary percolation as indicated by the solid line. It is also interesting to note that the probability of occurrence of a cluster size at the respective threshold values for different $\rho$ are almost same.

Two geometrical quantities of interest are studied here. One is the probability $P_{\infty}$ to find a lattice site in the spanning cluster and the other is the average cluster size $\chi$ excluding the spanning cluster. In terms of the area fraction $\mathrm{p}$, they are defined as $P_{\infty}=p-\Sigma^{\prime} s n_{\mathrm{s}}(p)$ and $\chi=$ $\Sigma^{\prime} \mathrm{s}^{2} n_{\mathrm{s}}(p) / \Sigma^{\prime} s n_{\mathrm{s}}(p)$. Therefore, in terms of $\rho$ and $g$ these quantities can be obtained as

$$
P_{\infty}=\rho+g(1-\rho)-\sum_{s}^{\prime} s n_{\mathrm{s}}(\rho, g)
$$

and

$$
\chi=\sum_{s}{ }^{\prime} s^{2} n_{\mathrm{s}}(\rho, g) / \sum_{s}{ }^{\prime} s n_{\mathrm{s}}(\rho, g)
$$

where the primed sum indicates that the spanning clus-

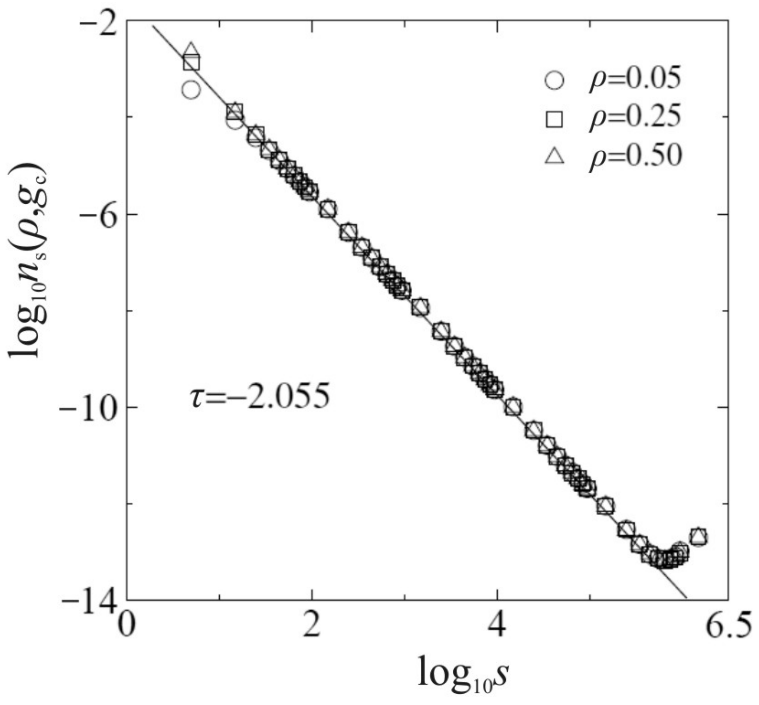

Figure 5. Plot of cluster size distribution per lattice site $n_{\mathrm{s}}$ against $s$ at the percolation threshold $g_{\mathrm{c}}(\rho)$ for three different $\rho$ values $0.05(\circ), 0.25(\square), 0.50(\Delta)$ for the lattice size $L=2048$. The exponent $\tau$ is found to be 2.055 .

ter is excluded. Their scaling behaviour are then determined by the first and second moment of the cluster size distribution function $n_{\mathrm{s}}(\rho, g)$ respectively. In general the $k$ th moment of the cluster size distribution can be obtained as

$$
\begin{aligned}
\left\langle s^{k}\right\rangle & =\int_{0}^{\infty} s^{k} n_{\mathrm{s}}(\rho, g) \mathrm{d} s \\
& =\int_{0}^{\infty} s^{k} f\left[\left(g-g_{\mathrm{c}}\right)(1-\rho) s^{\sigma}\right] \mathrm{d} s .
\end{aligned}
$$

Taking a new variable $t=\left(g-g_{\mathrm{c}}\right)(1-\rho) s^{\sigma}$ which also varies from 0 to $\infty$ for given $g$ and $\rho$ values, one could show that

$$
\left\langle s^{k}\right\rangle \approx\left[\left(g-g_{\mathrm{c}}\right)(1-\rho)\right]^{-(k-\tau+1) / \sigma}
$$

with a constant prefactor given by the value of the integral $\int_{0}^{\infty} t^{(k-\tau+1-\sigma) / \sigma} f(t) \mathrm{d} t$. . Then, taking $k=1$ one has,

$$
P_{\infty} \approx\left[\left(g-g_{\mathrm{c}}\right)(1-\rho)\right]^{\beta}
$$

where $\beta=(\tau-2) / \sigma$ and taking $k=2$ one has

$$
\chi \approx\left[\left(g-g_{\mathrm{c}}\right)(1-\rho)\right]^{-\gamma}
$$

where $=(3-\tau) / \sigma$.

For the system size $L=2048, P_{\infty}$ and $\chi$ are estimated for several values of $g$ above and below $g_{\mathrm{c}}(\rho)$ respectively for three different values of $\rho=0.05,0.25$, 

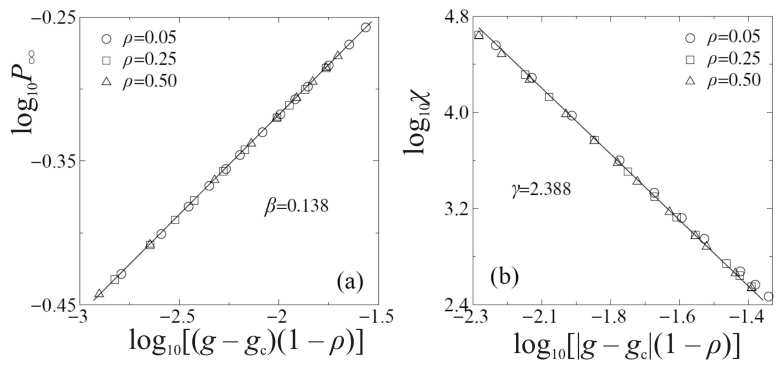

Figure 6. (a) Plot of $P_{\infty}$ versus $\left(g-g_{\mathrm{c}}\right)(1-\rho)$ for $g>g_{\mathrm{c}}(\rho)$ and (b) Plot of $\chi$ versus $\left|g-g_{\mathrm{c}}\right|(1-\rho)$ for $g<g_{\mathrm{c}}(\rho)$ for three different $\rho$ values $0.05(\circ), 0.25(\square), 0.50(\Delta)$ for $L=2048$.

0.50. In Figure 6a $P_{\infty}$ is plotted against $\left(g-g_{\mathrm{c}}\right)(1-\rho)$ for $g>g_{\mathrm{c}}(\rho)$ and in Figure $6 \mathrm{~b}, \chi$ is plotted against $\mid g-$ $g_{\mathrm{c}} \mid(1-\rho)$ for $g<g_{\mathrm{c}}(\rho)$. It can be seen that both $P_{\infty}$ and $\chi$ obey power law scaling with their respective critical exponents $\beta \approx 5 / 36$ and $\gamma \approx 43 / 18$ as that of percolation. The solid lines are guide to eye in these figures. Since the critical exponents and the fractal dimension are all that of ordinary percolation, the scaling relations among them such as $\beta=(\tau-2) / \sigma$ and $\gamma=(3-\tau) / \sigma$ all are satisfied. The existence of power law scaling indicates that the transition studied here is continuous or second order rather than discontinuous or first order. ${ }^{20}$

The scaling function form of $n_{\mathrm{s}}(\rho, g)=\mathrm{s}^{-\tau} f[(g-$ $\left.\left.g_{\mathrm{c}}\right)(1-\rho) s^{\sigma}\right]$ can be verified by plotting $n \mathrm{~s}(\rho, g) / n_{\mathrm{s}}\left(\rho, g_{\mathrm{c}}\right)$ against the scaled variable $\left(g-g_{\mathrm{c}}\right)(1-\rho) s^{\sigma}$. If $f(0)$ is a constant, it is expected that the plot of $n \mathrm{~s}(\rho, g) / n_{\mathrm{s}}\left(\rho, g_{\mathrm{c}}\right)$ against the scaled variable $\left(g-g_{\mathrm{c}}\right)(1-\rho) s^{\sigma}$ for different values of $\rho, g$ and $s$ should collapse onto a single curve for the right value of the exponent $\sigma$. In Figure 7, $n \mathrm{~s}(\rho$, $g) / n_{\mathrm{s}}\left(\rho, g_{\mathrm{c}}\right)$ is plotted against the scaled variable $(g-$ $\left.g_{\mathrm{c}}\right)(1-\rho) s^{\sigma}$ taking $\sigma=0.4$. It can be seen that a good data collapse is observed irrespective of the values of $\rho$, $g$ or $s$. Thus the value of $\sigma$ taken here (approximately the same value of $\sigma$ of percolation) is correct.

\section{FINITE SIZE SCALING}

The scaling form of different geometrical quantities measured are very often found to be limited by the finite system size $L$. A system is said to be finite if its size $L$ is less than the connectivity length $\xi$. If a quantity $Q$ is predicted to scale as $\left|p-p_{\mathrm{c}}\right|^{-q}$ for the system size $L>>\xi$, then the scaling form of $Q$ for the system size $L<<\xi$ is expected to be

$$
Q(p, L)=L^{q / v} f\left[\left(p-p_{\mathrm{c}}\right) L^{1 / v}\right]
$$

On substitution of the value of $\left(p-p_{c}\right)=\left(g-g_{c}\right)(1-\rho)$ in Eq. 19, one has

$$
Q(\rho, g, L)=L^{q / v} f\left[\left(g-g_{\mathrm{c}}\right)(1-\rho) L^{1 / v}\right]
$$

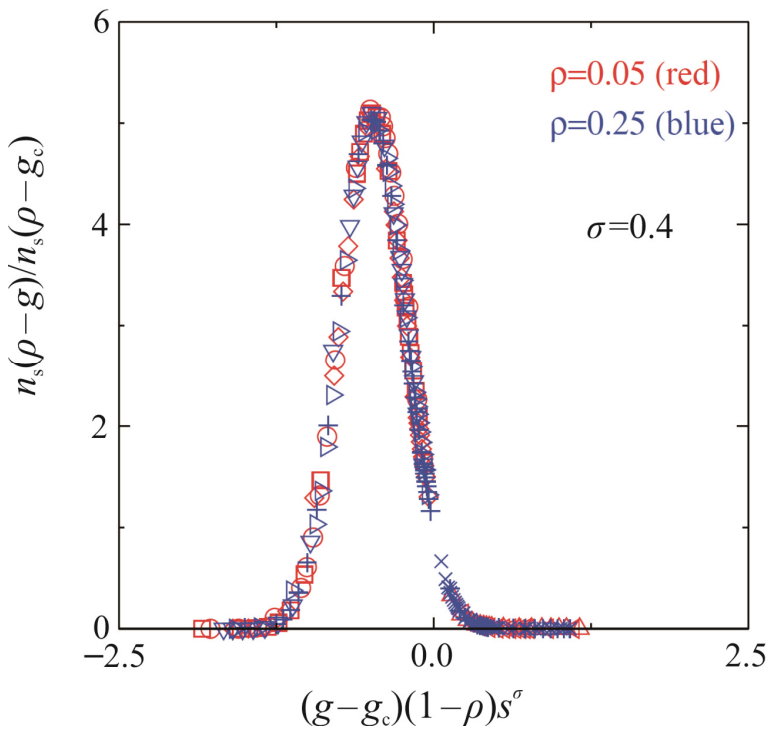

Figure 7. (Color online) Plot of $n_{\mathrm{s}}(\rho, g) / n_{\mathrm{s}}\left(\rho, g_{\mathrm{c}}\right)$ against ( $g-$ $\left.g_{\mathrm{c}}\right)(1-\rho) s^{\sigma}$. For $\rho=0.05$ (red), the growth probability $g$ is taken as $0.500(\circ), 0.530(\square), 0.550(\diamond), 0.650(\Delta), 0.700(\triangleright)$ and for $\rho=0.25$ (blue) the growth probabilities are $g=0.400(\nabla)$, $0.425(\triangleleft), 0.440(+), 0.500(\times), 0.550\left(^{*}\right)$. For $\sigma=0.4$, a good data collapse is obtained.

Therefore, the finite size scaling form of $P_{\infty}$ and the average size $\chi$ are expected to be

$$
\begin{aligned}
& P_{\infty}(\rho, g, L)=L^{-\beta / v} F\left[\left(g-g_{\mathrm{c}}\right)(1-\rho) L^{1 / v}\right] \\
& \chi(\rho, g, L)=L^{\gamma / v} G\left[\left(g-g_{\mathrm{c}}\right)(1-\rho) L^{1 / v}\right]
\end{aligned}
$$

since $P_{\infty} \sim\left(p-p_{\mathrm{c}}\right)^{\beta}$ and $\chi \sim\left|p-p_{\mathrm{c}}\right|^{-\gamma}$.

Both the quantities $P_{\infty}$ and are measured at $g=$ $g_{\mathrm{c}}(\rho)$ for different values of the system size $L$. At $g=$ $g_{\mathrm{c}}$, their scaling forms are expected to be $P_{\infty}(\rho, g, L) \approx$ $L^{-\beta / v}$ and $\chi(\rho, g, L) \approx L^{\gamma / v}$ assuming that both $F(0)$ and $G(0)$ are constants. $P_{\infty}$ and $\chi$ are plotted against $L$ for different $\rho$ values in Figure 8(a) and (b) respectively. It can be seen that they follow the respective scaling behaviours with exponents $\beta / v=0.104$ and $\gamma / v=1.79$
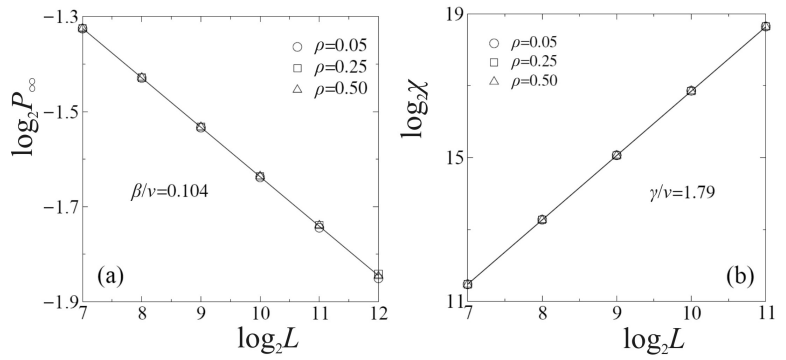

Figure 8. (a) Plot of $P_{\infty}$ against system size $L$ and (b) Plot of $\chi$ against system size $L$ at $g=g_{\mathrm{c}}(\rho)$ for $\rho=0.05(\circ), 0.25(\square) \&$ $0.50(\Delta)$. 


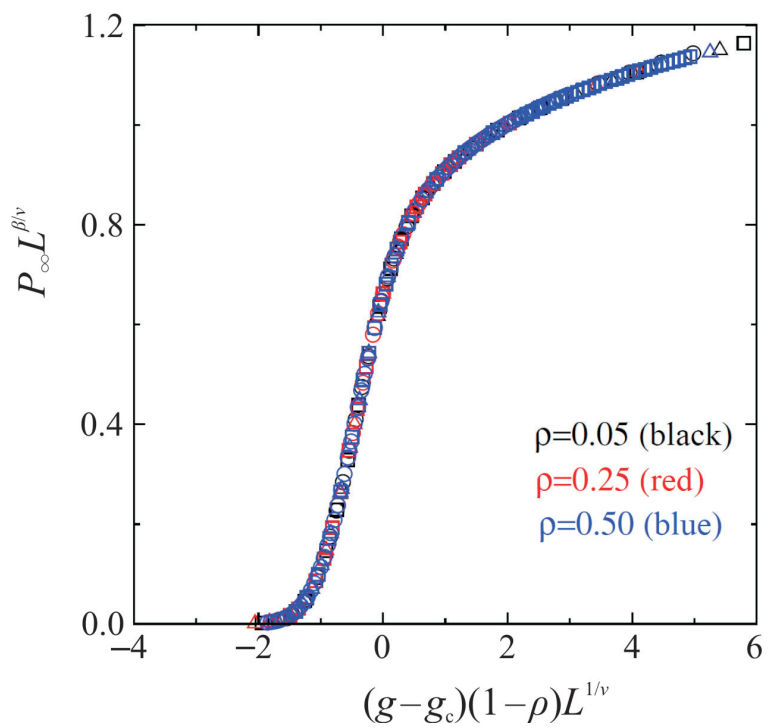

Figure 9. (Color online) Plot of $P_{\infty} L^{\beta / v}$ against $\left(g-g_{\mathrm{c}}\right)(1-$ $\rho) L^{1 / v}$ for $\rho=0.05$ (black), 0.25 (red), 0.50 (blue) and for $L=$ 512(०), 1024( $\square)$ and 2048( $\Delta$ ) for each $\rho$. A good data collapse is obtained for the estimated critical exponents.

respectively. The ratio of the exponents again confirms the percolation exponents. It is also interesting to note that the absolute values of these quantities are independent of the initial seed concentration $\rho$. Since by definition $P_{\infty}=S_{\max } / L^{2}$ and $S_{\max } \approx L^{d_{f}}$, it is expected that $P_{\infty}$ should scale as $L^{d_{f}-2}$. Hence one should have $d_{f}=2-\beta / v$. The scaling relation is exactly satisfied here because all critical exponents are as that of percolation.

The finite size scaling form of $P_{\infty}(\rho, g, L)=$ $L^{-\beta / v} F\left[\left(g-g_{\mathrm{c}}\right)(1-\rho) L^{1 / v}\right]$ is now verified. In Figure 9, $P_{\infty} L^{\beta / v}$ is plotted against the scaled variable $\left(g-g_{\mathrm{c}}\right)(1-$ $\rho) L^{1 / v}$ for three different values of $\rho$ and three different system sizes $L=512,1024 \& 2048$ for each $\rho$ taking $\beta / v$ and $1 / v$ as that of percolation. A good data collapse is observed for all values of $\rho$ and $L$. It can also be noted that no discontinuous transition is observed for any $\rho$ and $L$ values.

\section{SUMMARY AND CONCLUSION}

A generalized random cluster growth model is developed to study percolation transition. In this model two tunable parameters, the initial seed concentration $\rho$ and the cluster growth probability $g$, are kept. It is found that for each $\rho$ value there exists a critical growth probability $g_{\mathrm{c}}$ at which a continuous percolation transition occurs for the first time. It is identified that the values of the critical exponents are that of ordinary percolation and all such transitions belong to the same universality class of percolation. The results are also verified by finite size scaling analysis. A phase line consisting of second order phase transition points is found to separate the percolating region from the non-percolation region in the $\rho-g$ parameter space. No EP transition is expected in this model because no mechanism of suppression of growth of one cluster over the other depending on their sizes is present in this model.

The model could have application in the study of growth of micro-organisms in controlled conditions.

\section{REFERENCES}

1. P. R. King et al., Physica A 274 (1999) 60; Physica A 314 (2002) 103 (2002).

2. J. L. Cardy and P. Grassberger, J. Phys. A: Math. Gen. 18 (1985) L267.

3. R. Cohen, D. Ben-Avraham, and S. Havlin, Phys. Rev. E 66 (2002) 036113.

4. A. Acin, J. I. Cirac, and M. Lewenstein, Nature Physics 3 (2007) 256.

5. H. J. Herrmann and S. Roux, editors, Statistical Models for the Fracture of Disordered Media, North-Holland, 1990.

6. Z. Ball, H. M. Phillips, D. L. Callahan, and R. Sauerbrey, Phys. Rev. Lett. 73 (1994) 2099.

7. H. E. Roman, A. Bunde and W. Dieterich, Phys. Rev. B 34 (1986) 3439.

8. M. Sahimi, Applications of Percolation Theory, Taylor and Francis, London, 1994.

9. D. Stauffer and A. Aharony, Introduction to Percolation Theory, second edition, Taylor and Francis, London, Washington, DC, 1992.

10. D. Achlioptas, R. M. D'Souza, and J. Spencer, Science 323 (2009) 1453

11. R. M. Ziff, Phys. Rev. Lett. 103 (2009) 045701; Phys. Rev. E 82 (2010) 051105.

12. F. Radicchi and S. Fortunato, Phys. Rev. Lett. 103 (2009) 168701; Phys. Rev. E 81 (2010) 036110.

13. P. Grassberger, C. Christensen, G. Bizhani, S.-W. Son, and M. Paczuski, Phys. Rev. Lett. 106 (2011) 225701.

14. R. A. da Costa, S. N. Dorogovtsev, A. V. Goltsev, and J. F. F. Mendes, Phys. Rev. Lett. 105 (2010) 255701.

15. Y. S. Cho, S. Hwang, H. J. Herrmann, and B. Kahng, Science 339 (2013) 1185.

16. J. Hoshen and R. Kopelman, Phys. Rev. B 14 (1976) 8.

17. P. L. Leath, Phys. Rev. B 14 (1976) 5046.

18. Y. A. Andrienko, N. V. Brilliantov, and P. L. Krapivsky, J. Stat. Phys. 75 (1994) 507; N. V. Brilliantov, P. L. Krapivsky, and Y. A. Andrienkov, J. Phys. A 27 (1994) L381.

19. N. Tsakiris, M. Maragakis, K. Kosmidis, and P. Argyrakis, Phys. Rev. E 82 (2010) 041108; Eur. Phys. J. B 81 (2011) 303.

20. R. M. Ziff, Science 339 (2013) 1159 